\title{
Respective influence of in-plane and out-of-plane spin-transfer torques in magnetization switching of perpendicular magnetic tunnel junctions
}

\author{
A. A. Timopheev, R. Sousa, M. Chshiev, L. D. Buda-Prejbeanu, and B. Dieny \\ Univ. Grenoble Alpes, INAC-SPINTEC, F-38000 Grenoble, France; \\ CEA, INAC-SPINTEC, F-38000 Grenoble, France; \\ CNRS, SPINTEC, F-38000 Grenoble, France
}

(Received 3 June 2015; revised manuscript received 26 August 2015; published 28 September 2015)

\begin{abstract}
The relative contributions of in-plane (damping-like) and out-of-plane (field-like) spin-transfer torques (STT) in the magnetization switching of out-of-plane magnetized magnetic tunnel junctions (pMTJ) has been theoretically analyzed using the transformed Landau-Lifshitz-Gilbert (LLG) equation with the STT terms. It is demonstrated that in a pMTJ structure obeying macrospin dynamics, the out-of-plane torque influences the precession frequency, but it does not contribute significantly to the STT switching process (in particular to the switching time and switching current density), which is mostly determined by the in-plane STT contribution. This conclusion is confirmed by finite temperature and finite writing pulse macrospin simulations of the current field switching diagrams. It contrasts with the case of STT switching in in-plane magnetized magnetic tunnel junction (MTJ) in which the field-like term also influences the switching critical current. This theoretical analysis was successfully applied to the interpretation of voltage field STT switching diagrams experimentally measured on pMTJ pillars $36 \mathrm{~nm}$ in diameter, which exhibit macrospin behavior. The physical nonequivalence of Landau and Gilbert dissipation terms in the presence of STT-induced dynamics is also discussed.
\end{abstract}

DOI: 10.1103/PhysRevB.92.104430

PACS number(s): 85.75.-d, 75.78.-n, 85.70.Ay

\section{INTRODUCTION}

Fully perpendicular magnetic tunnel junctions (pMTJ) constitute the storage element of spin-transfer torque magnetoresistive random access memory (STT-MRAM) [1-6]. STTMRAMs are very promising emerging nonvolatile memories since they combine nonvolatility, low energy consumption, high thermal stability, and almost unlimited endurance. The strongest research and development efforts are nowadays focused on out-of-plane magnetized $\mathrm{MgO}$-based MTJs. Indeed, the latter combine several advantages. They exhibit a high tunnel magnetoresistance effect [7] amplitude due to a very efficient spin-filtering phenomenon associated with the symmetry of the tunneling electron wave function $[8,9]$. Furthermore, they present a very large perpendicular anisotropy at the interface between the magnetic electrode and the $\mathrm{MgO}$ oxide barrier (Ks $\sim 1.4 \mathrm{erg} / \mathrm{cm}^{2}$ ) [10], which allows the storage layer magnetization to achieve a quite high thermal stability and therefore long memory retention. Additionally, a remarkable property of this interfacial anisotropy is that it exists in materials having weak spin-orbit coupling and therefore relatively low Gilbert damping $\alpha(\alpha<0.01)$. This is very important in STT-MRAM since the critical current for STT-induced switching $[11,12]$ of storage layer magnetization is directly proportional to the Gilbert damping. The advantage of using out-of-plane rather than in-plane magnetized MTJs in STT-MRAM is twofold: first, the interfacial perpendicular anisotropy at the $\mathrm{CoFeB} / \mathrm{MgO}$ interface provides higher thermal stability at smaller dimensions (sub-60 nm) than does the usual-shaped anisotropy by giving elliptical shape to in-plane magnetized MTJs. Second, for a given retention, i.e., a given thermal stability factor, the critical current for STT-induced switching is lower with an out-of-plane than with an in-plane magnetized storage layer $[13,14]$.

From a theoretical point of view, a first approach to STT-induced switching can be developed by solving the
Landau-Lifshitz-Gilbert (LLG) equation under the assumptions of $0 \mathrm{~K}$ macrospin approximation under stationary applied spin-polarized current. The equilibrium configurations of the system can thus be calculated, and the precessional dynamics of the system submitted to a small perturbation from the static equilibrium can be studied. This allows derivation of the threshold current required to achieve STT switching, as was done in Refs. [13-15]. Thermal fluctuations can be taken into account in several limiting cases using the Fokker-Planck equation. Thermal activation mainly decreases the threshold current value and the switching time, introducing an undesirable stochastic magnitude effect in both parameters $[16,17]$. The influence of the writing pulse duration was also theoretically studied [16,18-21].

Despite the numerous experimental results $[22,23]$ and micromagnetic simulations [24-26] generally pointing to quantitative disagreements with the macrospin-based estimations, usage of the macrospin approach is still justified for at least two reasons. First, it gives a simple but solid picture of the physical processes involved in STT switching that creates a common basis for qualitative analysis of the different magnetic multilayered systems, while most of the conclusions derived from micromagnetic approaches are rather of particular character. Micromagnetic behavior can be mimicked, for example, by introduction of an effective activation volume instead of Stoner-Wohlfarth behavior, but still using a thermal activation model for the subvolume [22]. Second, considering the general trend to reduce the volume of the storage element (and, consequently, the energy needed per write/read cycle), magnetic memory elements will eventually behave in a macrospin manner.

Based on these viewpoints, we investigated STT switching in fully perpendicular magnetic tunnel junction systems, where in addition to the Slonczewski STT term (sometimes called parallel or in-plane torque since it lies in the plane defined 
by the local magnetization and that of the spin polarization usually defined by the magnetization direction of the reference pinned layer), having a damping-like structure, an out-of-plane (also called field-like or perpendicular) term exists. Several theoretical papers predicted that the torque produced by the out-of-plane STT term could reach an amplitude comparable to that of in-plane torque [27-29]. Several experimental papers carried out on in-plane MTJ structures have already estimated it to be in the range of $30-40 \%$ of the in-plane torque [30-33]. It was mentioned [34] that its presence may lead to a backswitching process, a very undesirable effect in magnetic memory applications causing write errors.

In this paper, after having analyzed the Landau-LifshitzGilbert-Slonczewski equation mathematically transformed into Landau-Lifshitz (LL) form, we show that in fully perpendicular MTJ structures, the field-like torque plays a negligible role in the switching process. In contrast to in-plane MTJ systems [30-34], it only influences the precessional frequency preceding the switching, but the switching current density is primarily determined by the in-plane STT term. The experiment carried out on 36-nm-diameter pMTJ pillar supports our conclusions.

\section{PHASE BOUNDARIES FROM LLG EQUATION TRANSFORMED INTO LL EQUATION}

The most accepted form of the LLG equation describing dynamics of a macrospin under constant spin-polarized current can be presented as

$$
\begin{aligned}
\frac{d \hat{\boldsymbol{m}}}{d t}= & -\gamma\left(\hat{\boldsymbol{m}} \times \overrightarrow{\boldsymbol{H}}_{\mathrm{eff}}\right)+\alpha\left(\hat{\boldsymbol{m}} \times \frac{d \hat{\boldsymbol{m}}}{d t}\right) \\
& -\gamma \hat{\boldsymbol{m}} \times\left(\hat{\boldsymbol{m}} \times a_{\|} \hat{\boldsymbol{p}}\right)+\gamma \hat{\boldsymbol{m}} \times a_{\perp} \hat{\boldsymbol{p}},
\end{aligned}
$$

where $\hat{\boldsymbol{m}}=\frac{\overrightarrow{\boldsymbol{M}}}{\mathrm{M}_{\mathrm{S}}}$ is the unit vector along the free layer magnetization direction (in which $\mathrm{M}_{\mathrm{S}}$ is the free layer's volume magnetization saturation parameter), $\overrightarrow{\boldsymbol{H}}_{\text {eff }}$ is the effective field (comprising applied field, anisotropy field, and demagnetizing field), $\hat{\boldsymbol{p}}$ is the unit vector along the magnetization direction of the polarized layer, $\alpha$ is Gilbert damping, $\gamma$ is the gyromagnetic ratio, and $a_{\|}$and $a_{\perp}$ are in-plane (damping-like) and out-ofplane (field-like) STT prefactors, respectively. Both prefactors can be phenomenologically represented as functions of spin polarization in the magnetic electrodes, current density, or voltage bias applied to the tunneling barrier, as will be done later in the text.

In-plane and out-of-plane STT terms as written in Eq. (1) are geometrically equivalent to the precession and damping terms of the LL equation. One can therefore transform Eq. (1) into LL form using the standard technique, i.e., by making an $\hat{\boldsymbol{m}} \times$ product on both sides of the equation,

$$
\begin{aligned}
\hat{\boldsymbol{m}} \times \frac{d \hat{\boldsymbol{m}}}{d t}= & -\gamma \hat{\boldsymbol{m}} \times\left(\hat{\boldsymbol{m}} \times \overrightarrow{\boldsymbol{H}}_{\mathrm{eff}}\right)+\alpha \hat{\boldsymbol{m}} \times\left(\hat{\boldsymbol{m}} \times \frac{d \hat{\boldsymbol{m}}}{d t}\right) \\
& -\gamma a_{\|} \hat{\boldsymbol{m}} \times[\hat{\boldsymbol{m}} \times(\hat{\boldsymbol{m}} \times \hat{\boldsymbol{p}})]+\gamma a_{\perp} \hat{\boldsymbol{m}} \times(\hat{\boldsymbol{m}} \times \hat{\boldsymbol{p}})
\end{aligned}
$$

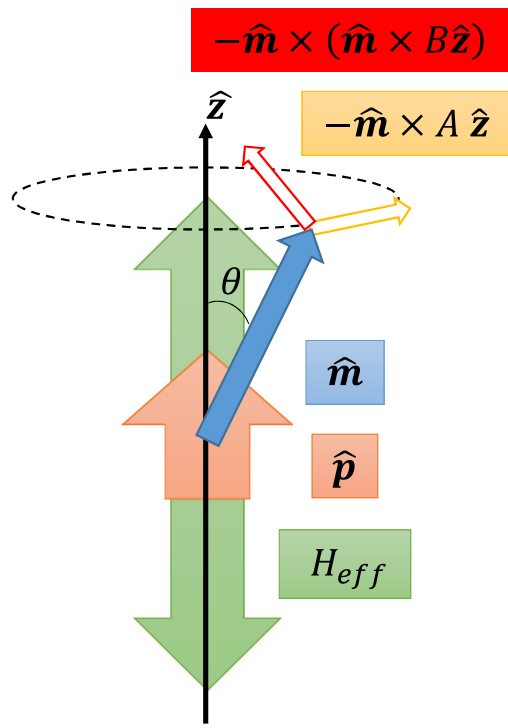

FIG. 1. (Color online) Geometry of the fully perpendicular MTJ system.

and replacing the damping term in Eq. (1) with the result. This yields,

$$
\begin{aligned}
\frac{\left(1+\alpha^{2}\right)}{\gamma} \frac{d \hat{\boldsymbol{m}}}{d t}= & -\hat{\boldsymbol{m}} \times\left[\overrightarrow{\boldsymbol{H}}_{\mathrm{eff}}-\left(a_{\perp}+\alpha a_{\|}\right) \hat{\boldsymbol{p}}\right] \\
& -\hat{\boldsymbol{m}} \times\left\{\hat{\boldsymbol{m}} \times\left[\alpha \overrightarrow{\boldsymbol{H}}_{\mathrm{eff}}-\left(\alpha a_{\perp}-a_{\|}\right) \hat{\boldsymbol{p}}\right]\right\}
\end{aligned}
$$

To this moment, all the transformations born only a character of mathematical identities, and Eq. (2) is valid for any system with any configuration of $\overrightarrow{\boldsymbol{H}}_{\text {eff }}$ and $\hat{\boldsymbol{p}}$. Rewritten in such a way, it acquires a more suitable form for further analytical treatment because dynamics in this system are fully determined by two vectors, namely $\left[\overrightarrow{\boldsymbol{H}}_{\text {eff }}-\left(a_{\perp}+\alpha a_{\|}\right) \hat{\boldsymbol{p}}\right]$ and $\left[\alpha \overrightarrow{\boldsymbol{H}}_{\mathrm{eff}}-\left(\alpha a_{\perp}-a_{\|}\right) \hat{\boldsymbol{p}}\right]$, which have many similarities and whose form can be significantly simplified as soon as the geometry of $\overrightarrow{\boldsymbol{H}}_{\text {eff }}$ and $\hat{\boldsymbol{p}}$ has been set. Also, the use of Eq. (2) is more convenient in numerical integration schemes. Further analysis will focus on the case of the pMTJ structure assuming macrospin dynamics of the storage layer described by Eq. (2).

We consider fully perpendicular magnetic tunnel junctions submitted to an out-of-plane external magnetic field $\overrightarrow{\boldsymbol{H}}_{\text {ext }}$ and, therefore, applied parallel to the symmetry axis. This situation allows analytical analysis wherein the quantities $\overrightarrow{\boldsymbol{H}}_{\mathrm{eff}}, \hat{\boldsymbol{p}}, \overrightarrow{\boldsymbol{H}}_{\mathrm{ext}}, \hat{z}$ remain collinear independently of the instantaneous direction of $\hat{\boldsymbol{m}}$. The magnetic free energy density functional $U$ of such a system depends only on one variable, $\theta$, the angle between magnetization vector $\hat{\boldsymbol{m}}$ and quantization axis $\hat{z}$ (see Fig. 1) and is written:

$$
U=\left(K_{\perp}-2 \pi M_{\mathrm{S}}^{2}\right) \sin ^{2} \theta-M_{\mathrm{S}} H_{\mathrm{ext}} \cos \theta .
$$

When $\left|H_{\text {ext }}\right|<H_{\perp}, \quad H_{\perp}=\frac{2 K_{\perp}}{M_{S}}-4 \pi M_{\mathrm{S}}$, and $H_{\mathrm{ext}}=$ $\overrightarrow{\boldsymbol{H}}_{\text {ext }} \cdot \hat{z}$, there are two stable magnetic moment orientations 
independent of $H_{\mathrm{ext}}$ and always collinear with $\hat{z}$ :

$$
\begin{aligned}
& \frac{\partial U}{\partial \theta}=0, \frac{\partial^{2} U}{\partial \theta^{2}}>0, \\
& -H_{\perp}<H_{\mathrm{ext}}<H_{\perp}, \rightarrow \theta_{0}=0, \quad \theta_{0}=\pi . \\
& H_{\perp}>0 .
\end{aligned}
$$

The collinearity of the four vectors $\overrightarrow{\boldsymbol{H}}_{\text {eff }}, \hat{\boldsymbol{p}}, \overrightarrow{\boldsymbol{H}}_{\text {ext }}, \hat{z}$ greatly simplifies Eq. (2), allowing it to work only with the magnitudes $a_{\perp}, a_{\|}$, and $H_{\text {eff }}$ :

$$
\begin{aligned}
\frac{\left(1+\alpha^{2}\right)}{\gamma} \frac{d \hat{\boldsymbol{m}}}{d t} & =-\hat{\boldsymbol{m}} \times A \hat{z}-\hat{\boldsymbol{m}} \times(\hat{\boldsymbol{m}} \times B \hat{z}), \\
A & =H_{\mathrm{eff}}-\left(a_{\perp}+\alpha a_{\|}\right), \\
B & =\alpha H_{\mathrm{eff}}-\left(\alpha a_{\perp}-a_{\|}\right), \\
H_{\mathrm{eff}}=\overrightarrow{\boldsymbol{H}}_{\mathrm{eff}} \cdot \hat{z} & =-\frac{\partial U}{\partial \overrightarrow{\boldsymbol{M}} \cdot \hat{z}}=H_{\perp}\left(\cos \theta_{0}+H_{\mathrm{ext}} / H_{\perp}\right) .
\end{aligned}
$$

Here, two scalar parameters $A$ and $B$ are introduced, which represent the direction and magnitude of the perpendicular and in-plane (the plane is formed by $\hat{\boldsymbol{m}}$ and $\hat{\boldsymbol{p}}$ ) effective torques (see Fig. 1) acting on magnetization when the latter departs from its equilibrium position $\theta_{0}(0$ or $\pi)$ because of thermal fluctuations.

An important specific of the considered system is that the $A$ parameter cannot change orbit (i.e., the angle $\theta$ ); rather, it only influences the frequency of the precession. One can derive the ferromagnetic resonance (FMR) condition, which is just a modified "easy axis" Kittel formula for this case:

$$
\omega / \gamma=H_{\text {eff }}-\left(a_{\perp}+\alpha a_{\|}\right),
$$

where $\omega$ is the angular frequency of the resonance precession. One can see, that if $a_{\perp}>H_{\text {eff }}-\alpha a_{\|}$, the precession direction will be changed, while an increase or decrease of $\theta$ is exclusively determined by the sign of the $B$ parameter, wherein the damping-like STT term is dominating since $\alpha$ is usually small (typically in the range $0.007-0.02$ ). The precessional response of the system before the switching could be measured-for instance, by measuring $\omega$ versus the dc applied voltage bias $V_{\text {bias }}$ on a single pMTJ pillar either by rf voltage frequency detection, noise measurements [35], STT experiments, or microfocused Brillouin light scattering (BLS) FMR technique. The excitation frequency would give access to $a_{\perp}\left(V_{\text {bias }}\right)$ dependence, while the FMR linewidth parameter change versus $V_{\text {bias }}$ would reflect mostly $a_{\|}\left(V_{\text {bias }}\right)$ dependence.

Turning back to the analysis of Eq. (5) and Fig. 1, one can note that only the damping term, $\hat{\boldsymbol{m}} \times(\hat{\boldsymbol{m}} \times B \hat{z})$, can change the precession angle $\theta$. It is therefore possible to derive the boundary conditions for a current-magnetic field stability phase diagram. The magnetization switching process starts when the $B$ parameter changes sign. This condition yields the threshold criterion for the STT-induced magnetization switching:

$$
\alpha H_{\text {eff }}+a_{\|}-\alpha a_{\perp}=0 .
$$

One can see from Eq. (7) that the contribution from the in-plane STT term $\left(a_{\|}\right)$is largely dominating the switching process. Indeed, the in-plane torque is on the order of $\alpha H_{\text {eff }}$ while the contribution of the perpendicular torque is weighted by the Gilbert damping, resulting in a much weaker influence in the switching process. Here one can note again that the best method to determine $a_{\perp}$ experimentally is through FMR measurements, and not from the influence of $a_{\perp}$ on the (current, field) phase diagram boundaries since the latter is very weak. Indeed, from the above discussion, being able to see an influence of $a_{\perp}$ on the phase diagram boundaries would require $a_{\perp} \approx a_{\|} / \alpha$ which seems to be physically unachievable in standard pMTJ systems [27-34]. Also, as will be shown in Sec. VI, the $\alpha a_{\perp}$ term in Eq. (7) disappears if one chooses the dissipation term in the LL formulation. In any case, Eqs. $(6,7)$ are quite useful for the analysis of STT switching experiments performed on pMTJ systems.

\section{STABILITY PHASE DIAGRAM BOUNDARIES}

Having set the relations between electric current flowing through pMTJ and the STT prefactor magnitudes, one can construct the stability phase diagram explicitly from Eq. (7) assuming that the spin-polarized current pulse is long enough to complete any STT-induced switching while influence of the thermal fluctuations is limited to setting a small initial misalignment angle $\theta_{0}$ so that $\left|\cos \theta_{0}\right| \approx 1$. Modification of the phase boundaries due to thermal fluctuations and under a short-pulse writing regime, which are essential in real magnetic memory applications, will be analyzed in the following sections, while in this section, the conditions of long-pulse and low-temperature regime are assumed.

In most investigated pMTJs, one can expect the condition $a_{\perp}<a_{\|}$and $\alpha a_{\perp} \ll a_{\|}$to be fulfilled. In this case, one can set $a_{\perp}=0$ and build up the boundaries of the (current, field) stability phase diagram. In absence of the spin-polarized current ( $a_{\|}=0, a_{\perp}=0$ ), switching occurs when $\alpha H_{\text {eff }}$ changes sign, i.e., when $H_{\mathrm{ext}}=-H_{\perp}$ for $\theta_{0}=0$ and $H_{\mathrm{ext}}=H_{\perp}$ for $\theta_{0}=\pi$. This defines the vertical boundaries of the diagram shown in Fig. 2(a), depicted by dashed vertical lines. For $H_{\text {ext }}=0$ and by setting $a_{\|}=s_{t \|} G_{p} V_{\text {bias }}$ [where $s_{t \|}=\frac{\hbar}{2 e} \cdot \frac{\eta}{t_{\mathrm{F}} M_{\mathrm{S}}}=\mathrm{STT}$ is the conversion efficiency factor in units of $\mathrm{Oe} /\left(\mathrm{A} \mathrm{cm}^{-2}\right) ; \eta$ is the effective spin polarization parameter; and $G_{p}$ is the tunneling conductance factor, generally dependent on $\theta$ and $V_{\text {bias }}$, in units of $\Omega^{-1} \mathrm{~cm}^{-2}$, representing in the simplest interpretation the inverse of the $\mathrm{R} \times \mathrm{A}$ product], one can obtain that the switching current density $I_{\mathrm{sw}}$ is proportional to $\alpha H_{\perp}$ :

$$
I_{\mathrm{sw} 0}=G_{p} V_{\mathrm{sw} 0}=\frac{\alpha H_{\perp}}{s_{t \|}}=\frac{2 e}{\hbar} \cdot \frac{t_{\mathrm{F}} \alpha H_{\perp} M_{\mathrm{s}}}{\eta} .
$$

In the case of $H_{\text {ext }} \neq 0$, relation (8) leads to a linear dependence between the switching current and external magnetic field, yielding a linear slope on the switching phase diagram given by

$$
\frac{d I_{\mathrm{sw}}}{d H_{\mathrm{ext}}}=\frac{\alpha}{s_{t \|}}=\frac{2 e}{\hbar} \cdot \frac{t_{\mathrm{F}} \alpha M_{\mathrm{s}}}{\eta} .
$$

One can conclude that if the effective spin polarization parameter $\eta$ is constant (i.e., weakly dependent on the bias voltage $V_{\text {bias }}$ ), then the STT-driven parts of the switching diagram are linearly dependent on the applied field, with the slope 

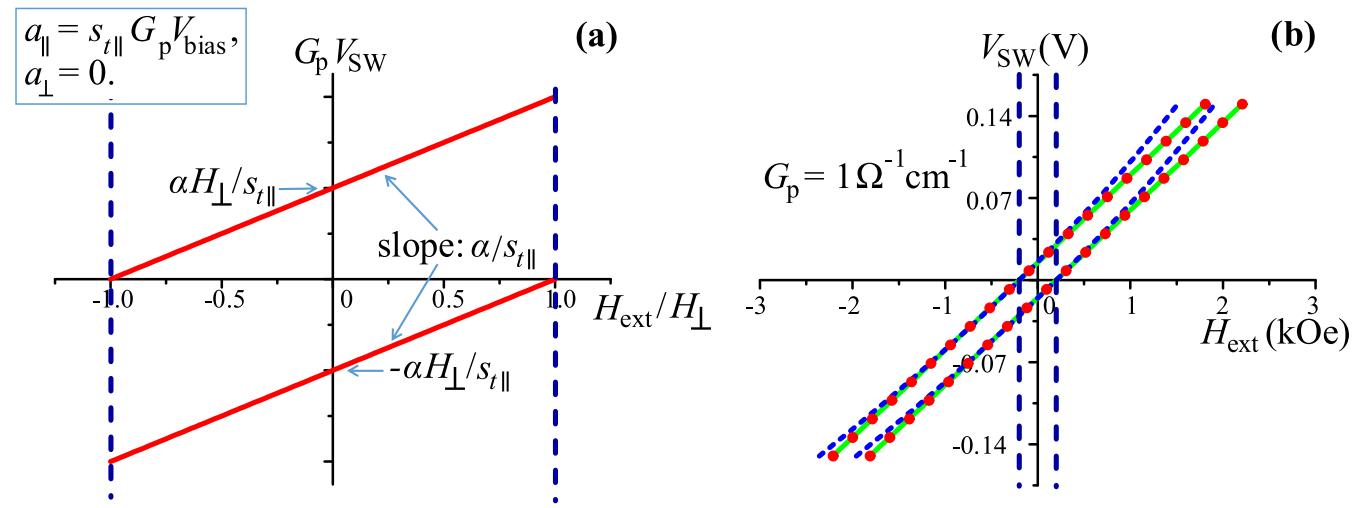

FIG. 2. (Color online) (a) Stability phase diagram constructed from Eq. (7) assuming $a_{\|}=s_{t \|} G_{p} V_{\text {bias }}$ and $a_{\perp}=0$; (b) modification of the phase boundaries for the same $a_{\|}$prefactor $\left(a_{\|}=s_{t \|} G_{p} V_{\text {bias }}, s_{t \|}=67 G_{p}^{-1} \mathrm{Oe} / \mathrm{V}\right)$ and different forms of $a_{\perp}$ prefactor: solid line $a_{\perp}=0$; circles $a_{\perp}=s_{t \perp 2}\left(G_{p} V_{\text {bias }}\right)^{2}$ with $s_{t \perp 2}=154 G_{p}^{-2} \mathrm{Oe} / \mathrm{V}^{2}$; dashed line $a_{\perp}=s_{t \perp 1} G_{p} V_{\text {bias }}+s_{t \perp 2}\left(G_{p} V_{\text {bias }}\right)^{2}$ with $s_{t \perp 1}=500 G_{p}^{-1}$ Oe $/ \mathrm{V}$ and $s_{t \perp 2}=$ $10000 G_{p}^{-2} \mathrm{Oe} / \mathrm{V}^{2}$. Other system parameters are $\alpha=0.05$ and $H_{\perp}=200 \mathrm{Oe}$.

proportional to the intrinsic damping parameter $\alpha$ and inversely proportional to the STT efficiency prefactor $s_{t \|}$, and with the zero-field switching current magnitude being proportional to the effective perpendicular anisotropy $H_{\perp}$. One should also note that Eq. (8) is in full agreement with previously obtained expressions [13-15,36] for the zero-field-threshold switching current derived from analysis of the precessional response of the system, assuming linear dependence of the damping-like STT prefactor versus the applied current. In our case, Eq. (7) allows one to calculate $I-H$ stability phase diagram boundaries for any $a_{\|}, a_{\perp}$ prefactors with arbitrary bias current (voltage) dependence, or by choosing it from the theoretical estimations made for the concrete MTJ system [28,29].

Simultaneous influence of both in-plane and out-of-plane STT terms on the phase boundaries is shown in Fig. 2(b). We have chosen realistic values for the magnetic system (see the figure caption), letting the in-plane prefactor be linearly dependent on bias voltage with $s_{t \|}=67 G_{p}^{-1} \mathrm{Oe} / \mathrm{V}$. As for the out-of-plane prefactor $a_{\perp}$, we show three different cases: zero, quadratic dependence with $s_{t \perp 2}=154 G_{p}^{-2} \mathrm{Oe} / \mathrm{V}^{2}$, and quadratic + linear dependence (which mimics features of an asymmetric MTJ structure; see the expression in the caption of Fig. 2) with unreasonably large STT conversion coefficients. One can see that within the difference between the phase boundaries in all three cases is negligible. The second case uses exactly the same parameters as those in Ref. [15] in Fig. 3. We can see that the boundaries calculated and simulated there are identical to our three cases, no matter what prefactor dependence is introduced $-H_{\perp}<$ $H_{\text {ext }}<H_{\perp}$, for the out-of-plane STT term. This confirms that the out-of-plane STT term has a negligible influence on the STT switching diagram. Parabolic shape of boundaries starts being observed only in the third case, and it becomes noticeably different only for current magnitudes several times larger than the threshold switching current. Thus, one can conclude that under long-pulse/low-temperature conditions, STT switching in fully perpendicular MTJ structures obeying macrospin dynamics is almost not influenced by the out-ofplane STT term and by its prefactor bias voltage or current dependence. Below, we will show that this statement is still valid at finite temperature and reasonably short writing pulses.

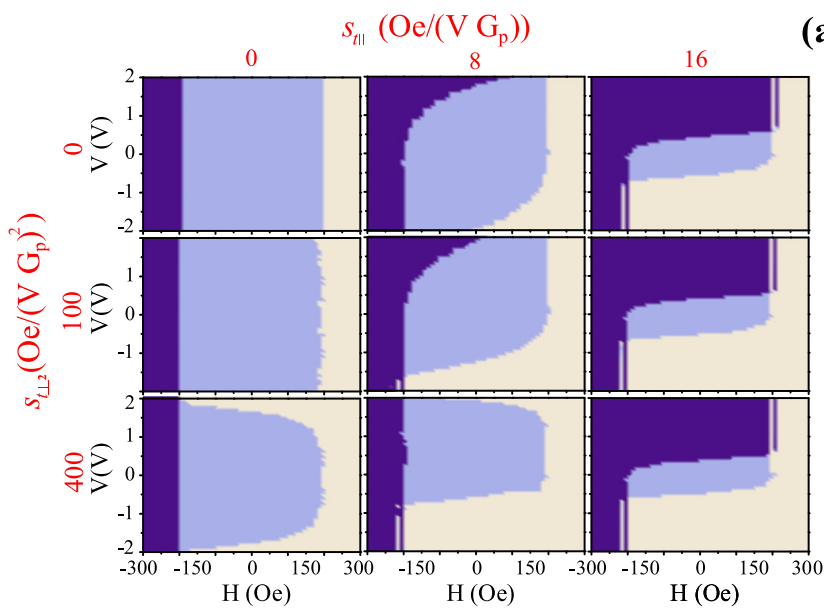

(a)

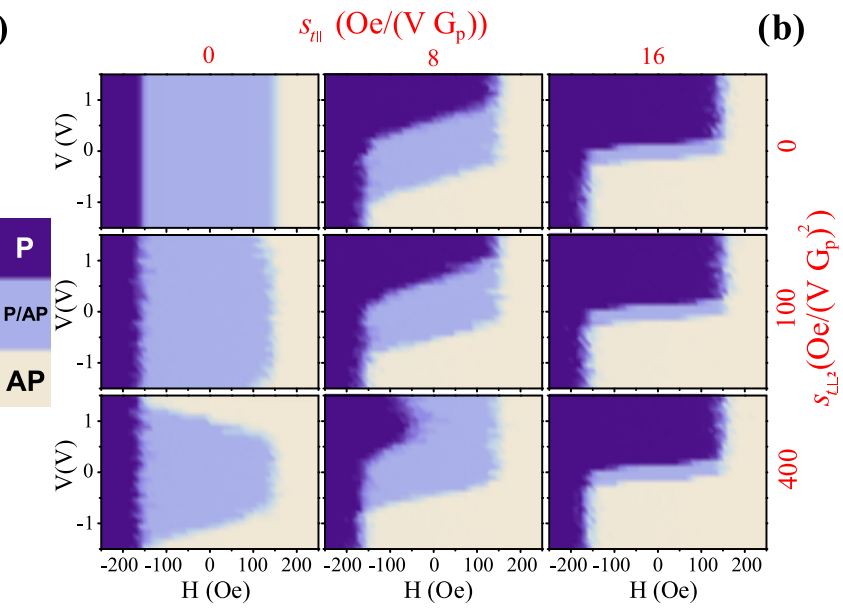

FIG. 3. (Color online) Finite writing pulse phase diagrams for different in-plane and out-of-plane STT prefactor magnitudes: (a) $T=0$ K; (b) $T=300 \mathrm{~K}$. The model parameters are $H_{\perp}=200 \mathrm{Oe}, \mathrm{g}=2.20$ (g-factor), $\alpha=0.01$. Integration time was $1 \mu \mathrm{s}$ in each field point, and the writing pulse width is $40 \mathrm{~ns}$. Each diagram is an average of 10 identical simulations. 


\section{MACROSPIN SIMULATIONS}

Aiming at extending the conclusions made in the previous sections to the case of finite temperatures and finite writing pulse regime, a series of macrospin simulations were performed using Eq. (2) (i.e., with Gilbert damping). The simulations were carried out with a fixed writing pulse duration of $40 \mathrm{~ns}$ and a cumulative integration time of $1 \mu$ s for each field point. The following assumptions of bias voltage dependences for the STT prefactors were used: $a_{\perp}=s_{t \perp 2} G_{p}^{2} V_{\text {bias }}^{2}$ and $a_{\|}=$ $s_{t \|} G_{p} V_{\text {bias }}$, which is the case of symmetrical MTJ systems with a high spin polarization parameter. For convenience, the parameter $G_{p}$ was set constant and equal to $1 \Omega^{-1} \mathrm{~cm}^{-2}$. The temperature was included in the form of a stochastic thermal field $\boldsymbol{H}_{\mathrm{th}}$ with Gaussian distribution [37], added directly to the effective field $H_{\text {eff }}$. Statistical properties of these thermal fluctuations are given by the following relations:

$$
\left\langle H_{\mathrm{th}, i}(t)\right\rangle=0
$$

and

$$
\left\langle H_{\mathrm{th}, i}(t) H_{\mathrm{th}, j}\left(t^{\prime}\right)\right\rangle=\frac{2 \alpha k_{\mathrm{B}} T}{\gamma M_{\mathrm{S}} V_{p}} \delta_{i j} \delta\left(t-t^{\prime}\right)
$$

where $k_{\mathrm{B}}$ is the Boltzmann constant, and $V_{p}$ is the free layer volume. The chosen LLG equation is integrated with a (predictor-corrector) Heun scheme [38]. Here we used $V_{p}=2.07 \times 10^{-17} \mathrm{~cm}^{3}, H_{\perp}=200 \mathrm{Oe}, M_{\mathrm{s}}=$ $1000 \mathrm{emu} / \mathrm{cm}^{3}$, which gives the effective stability factor at $T=300 \mathrm{~K}$ :

$$
\Delta=\frac{H_{\perp} M_{\mathrm{S}} V_{p}}{2 k_{\mathrm{B}} T}=50 .
$$

This set of the parameters was chosen to mimic working conditions of an actual STT-MRAM device. Two sets of macrospin simulations, at $T=0 \mathrm{~K}$ and $T=300 \mathrm{~K}$, respectively, presented in Fig. 3 show how the phase boundaries are changed for the different combinations of in-plane and out-of-plane STT prefactor magnitudes. We will discuss first the results shown in Fig. 3(a) corresponding to the case with finite pulse duration and no thermal fluctuations $(T=0 \mathrm{~K})$.

The finite duration of the writing pulse brings two main effects. First, the STT-driven boundaries are shifted toward much higher voltages (currents). Evidently, to achieve switching within the considered finite time period, one has to apply higher amplitudes for the writing pulses. In the initial stage, when $\hat{\boldsymbol{m}}$ is almost collinear with the symmetry axis $\hat{z}$, the torque is very weak, which results in very slow STT-induced dynamics in the system. It is evident that in absence of thermal fluctuations, the switching time from $\hat{\boldsymbol{m}} \| \hat{z}$ initially would be infinite for any spin-polarized current magnitude $[13,14]$. To avoid this in the $T=0 \mathrm{~K}$ simulations, a small misorientation $\left(0.1^{\circ}\right)$ between $\hat{\boldsymbol{p}}$ and $H_{\text {ext }}$ was introduced in the system. The second effect is nonlinearities of the phase boundaries, which are seen even on the diagrams with the in-plane STT term only. This effect is linked with a nonlinear dependence of time necessary for STT switching versus the applied magnetic field. Both effects are entirely of dynamical nature, and their influence on the phase boundaries can be theoretically described using the formalism developed in Ref. [16]. Renormalization of the effective dynamic time allows one to link dependence between the critical current, pulse width, and finite temperature. This also will be done in the next section, while here the discussion will be focused on a qualitative analysis of the relative contributions of the in-plane and out-of-plane STT terms to phase boundaries shapes.

One can see from Fig. 3(a) that the general behavior of the phase boundary modifications on the simulated phase diagrams under finite writing pulse regime is in agreement with the conclusions made in the previous sections for the $\mathrm{dc}$ regime. For the case of $s_{t \perp 2}=400 G_{p}^{-2} \mathrm{Oe} / \mathrm{V}^{2}$ and $s_{t \|}=$ $0 G_{p}^{-1} \mathrm{Oe} / \mathrm{V}$, the simulated phase diagram demonstrates a unidirectional STT switching due to quadratic dependence of $a_{\perp}$ versus applied voltage. In other words, switching to the antiparallel configuration is possible only for $s_{t \perp 2}>0$, $s_{t \|}=0$. Zero-field $\left(H_{\text {ext }}=0\right)$ STT switching voltage for this diagram is $\pm 1.6 \mathrm{~V}$. This voltage induces an effective STT field in the damping term of Eq. (2) of $~ 1000 \mathrm{Oe}$, which is five times higher than the effective perpendicular anisotropy field $H_{\perp}=200 \mathrm{Oe}$. At the same time, if one adds a relatively small damping-like prefactor $s_{t \|}=30 G_{p}^{-1} \mathrm{Oe} / \mathrm{V}$, it completely removes any apparent influence of the field-like STT term from the phase diagram, despite the huge value chosen for its prefactor. When the effective contributions from both prefactors are comparable, the phase diagram acquires a noticeable asymmetry, as can be seen for the last two diagrams in the middle column. However, such a combination of $s_{t \|}$ and $s_{t \perp 2}$ already can be physically unrealistic.

Figure 3(b) shows the same set of simulations made under $T=300 \mathrm{~K}$. Several temperature-induced effects are observed: (i) decrease of the coercive field showing that thermally activated magnetization reversal takes place when the external magnetic field substantially lowers the effective barrier height in the system; (ii) shift of the voltage-driven parts of the boundaries toward lower switching voltages. Thermal fluctuations of the magnetic moment direction increases the probability of launching STT switching thanks to a thermally induced misorientation between $\hat{\boldsymbol{m}}$ and $\hat{\boldsymbol{p}}$. This increases the initial STT amplitude and substantially decreases the switching time for a given writing pulse amplitude. This is consistent with earlier observations in STT-MRAM cells and with theoretical expectation of a $I_{\mathrm{c}}=I_{\mathrm{c} 0}\left\{1-\frac{k_{\mathrm{B}} T}{\Delta E} \ln \left(\frac{\tau}{\tau_{0}}\right)\right\}$ dependence of switching current on the pulse duration under finite temperature [39]. Therefore, Fig. 3(b) Indicates that the general features observed in the switching phase diagram at 0 K [i.e., Fig. 3(a)] are conserved at finite temperature and illustrates again the negligible role of the out-of-plane STT term in the switching process [see in particular the last column in Fig. 3(b)].

\section{EXPERIMENTAL MEASUREMENTS OF THE $(I-H)$ SWITCHING DIAGRAM}

In this section, the STT efficiency and other magnetic parameters of pMTJ pillars are directly extracted from the measured diagram. Nominal 50-nm-diameter pMTJ pillars were fabricated from an MTJ stack grown by magnetron sputtering. The stack contains a 1.7-nm-thick $\mathrm{Co}_{20} \mathrm{Fe}_{60} \mathrm{~B}_{20}$ free layer sandwiched between two $\mathrm{MgO}$ barriers. Magnetization saturation parameter of the free layer was measured to be $1030 \mathrm{emu} / \mathrm{cm}^{3}$. Current in-plane magnetotransport measurements (CIPTMR) yielded $\mathrm{R} \times \mathrm{A}=5.7 \Omega \mu \mathrm{m}^{2}$ and 
(a)
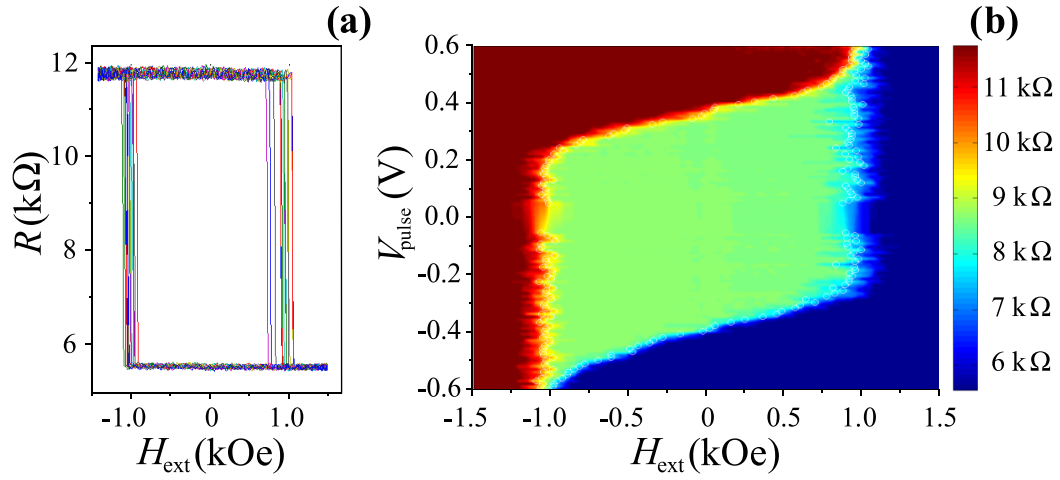

(b)

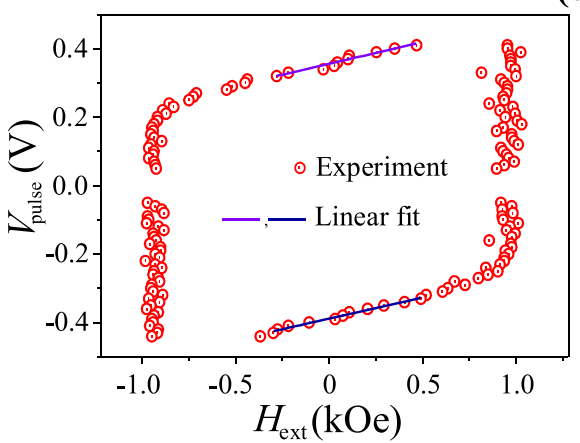

FIG. 4. (Color online) Experiment carried out on pMTJ pillar at room temperature applying 100-ns writing pulses. (a) Examples of magnetoresistance loops measured with zero writing pulses; (b) stability phase diagram; (c) extracted phase boundaries and their linear fittings.

$\mathrm{TMR}=126 \%$. The second $\mathrm{MgO}$ barrier was introduced to increase the perpendicular anisotropy of the free layer. It has a negligible resistance-area $(\mathrm{R} \times \mathrm{A})$ product compared with the main tunnel barrier. The bottom fixed layer is a synthetic antiferromagnetic-based, perpendicularly magnetized multilayer, and the polarizer material has the same composition as the free layer. The metallic electrode above the second $\mathrm{MgO}$ barrier is nonmagnetic. Experimentally, it was found that the pillar diameter slightly differs from its nominal value due to the nanofabrication technology $(36 \mathrm{~nm}$ instead of $50 \mathrm{~nm}$ nominal). This was recalculated using the values of the low resistive state $\left(R_{\mathrm{pp}}=5.6 \mathrm{k} \Omega\right.$ ) of the magnetoresistance curve [Fig. 4(a)] and assuming that the $\mathrm{R} \times \mathrm{A}$ value is preserved after the nanofabrication. Knowing the volume of the free layer in the pillar $V_{p}$, its room temperature coercivity, measurement time $(\sim 1 \mathrm{~s})$, and attempt frequency $f_{0}=10^{10} \mathrm{~s}^{-1}$, one can recalculate the perpendicular magnetic anisotropy from the Néel-Brown formula [37,40],

$$
H_{\mathrm{C}}(T)=H_{\perp}\left(1-\sqrt{\frac{2 k_{\mathrm{B}} T \ln \left(t_{m} f_{0}\right)}{M_{\mathrm{S}} H_{\perp} V_{p}}}\right),
$$

which gives $H_{\perp}=2.6 \mathrm{kOe}$ and $\Delta=56$.

The phase diagram measured at room temperature is shown in Fig. 4(b). At each magnetic field point, a 100-ns writing pulse with fixed amplitude was applied to the pMTJ pillar. Subsequently, the resistance was measured under small dc bias current, and the next magnetic field point was set. To reduce the stochasticity in the switching field values, the magnetoresistance loop was measured 15 times, and their average was used for switching field determination. The same procedure was used for all writing pulse amplitudes, and the final phase diagram was constructed from these averaged magnetoresistance loops. Magnetic field loop repetition frequency was $2 \mathrm{~Hz}$.

The extracted phase boundaries are shown in Fig. 4(c). The coercive field of the free layer is $940 \mathrm{Oe}$, and the coupling field with the reference layer is only $11 \mathrm{Oe}$ and is ferromagnetic. The voltage driven parts are linear and almost parallel to each other. To reduce the influence of small nonlinearities at the edges of the boundaries, only the central parts (within the \pm 500 Oe region) were used in the fitting. The extracted slopes are $1.27 \times$ $10^{-4}$ and $1.23 \times 10^{-4} \mathrm{~V} / \mathrm{Oe}$; their difference is within the fitting error. The zero-field switching voltages are 0.359 and
$0.385 \mathrm{~V}$, respectively. The difference is most probably due to the small dc bias current used for the resistance measurements.

The phase diagram shape is similar to those obtained from the theoretical analysis (Sec. III) and the simulations (Sec. IV) where the out-of-plane STT term is not dominating. For this system, we can choose the STT prefactor model $a_{\perp}=0, a_{\|}=$ $s_{t \|} G_{p} V_{\text {bias }}$. It corresponds to the dc diagram shown in Fig. 2 whose boundaries are described by Eqs. $(8,9)$. To recalculate the $s_{t \|}$ parameter from the extracted diagram slopes, one first needs to remap the experimental finite temperature-finite writing pulse diagram to that of the long pulse-low temperature model case. Here, we will follow the formalism described in Ref. [16]. Thermal effects in our case can be reduced to the regime of thermally assisted ballistic STT switching. In this regime, the main role of thermal fluctuations is to increase the probability of STT switching thanks to an increased initial misorientation angle $\theta_{0},\left|\cos \left(\theta_{0}\right)\right| \neq 1$. As already mentioned, STT switching dynamics starting from a tilted state reduces the switching time $\tau$, in agreement with $[13,14]$. The cone angle $2 \theta_{0}$, for which the equilibrium probability for the magnetic moment orientation distribution is 0.5 , is determined by thermal stability parameter $\Delta$ and applied magnetic field $\theta_{0}=(\ln 2 / \Delta)^{1 / 2}\left(1+H_{\text {ext }} H_{\perp}\right)^{-1 / 2}$, while the final angle, the extremum on the energy barrier $\theta_{\tau}=\arccos \left(-H_{\text {ext }} / H_{\perp}\right.$ ) (for $\theta_{0}<\pi / 2$ ), is determined by magnetic field (see Eq. (77) in Ref. [16]). Having defined the initial $\theta_{0}$ and final $\theta_{\tau}$ angles of the STT-induced dynamics, one can calculate analytically the switching time $\tau$ (see Eq. (58) in Ref. [16]):

$$
(i-1) \frac{\tau}{\tau_{\mathrm{D}}}=\ln \left(\frac{x_{\tau}}{x_{0}}\right)-\frac{1}{i+1} \ln \left(\frac{\frac{i-1}{i+1}+x_{\tau}^{2}}{\frac{i-1}{i+1}+x_{0}^{2}}\right),
$$

Here, $x_{0}=\tan \theta_{0}, x_{\tau}=\tan \theta_{\tau}, \tau_{\mathrm{D}}=\frac{\left(1+\alpha^{2}\right)}{\alpha \mu_{0} \gamma H_{\perp}}$, and, according to our formalism, $i=I_{\mathrm{sw}}^{\tau} / I_{\mathrm{sw} 0}-\frac{H_{\mathrm{ext}}}{H_{\perp}}$. Having calculated $\theta_{0}=6^{\circ}$ and $\tau_{\mathrm{D}}=9.9 \mathrm{~ns}$ and assuming $\alpha=0.02$ [41] and writing pulse duration $\tau=100 \mathrm{~ns}$, we recalculated $I_{\text {sw }}^{\tau}\left(H_{\text {ext }}\right)$ dependence from Eq. (11) (Fig. 5, blue line) and compared it with the $I_{\mathrm{sw}}\left(H_{\mathrm{ext}}\right)$ dc diagram case (Fig. 5, circles) derived from Eqs. $(8,9)$. One can conclude that 100 -ns writing pulses are long enough to remove the effect of dynamical distortion of the phase boundaries. For the measured device of Fig. 5, we find $\frac{\tau}{\tau_{\mathrm{D}}}=100.6$, which is quite high. This makes it possible to work directly with the phase boundaries [Eqs. $(8,9)]$ derived from 


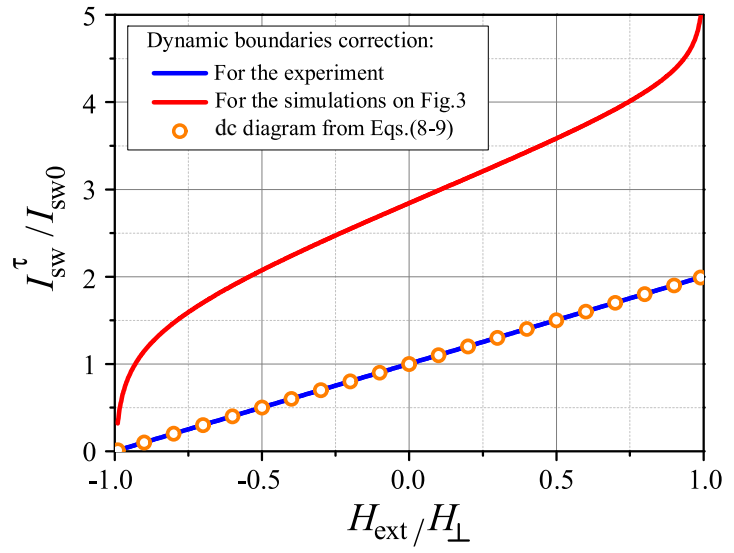

FIG. 5. (Color online) Finite pulse-finite temperature diagram boundary for $\frac{\tau}{\tau_{\mathrm{D}}}=100.6$ (blue for experiment) and $\frac{\tau}{\tau_{\mathrm{D}}}=1.5$ (red for simulations). The dots are the respective boundary obtained from Eqs. $(8,9)$.

Eq. (7). However, if $\frac{\tau}{\tau_{\mathrm{D}}}<10$ (the writing pulse width in the experiment would be $<10 \mathrm{~ns}$ ) and/or $\theta_{0}$ is too small, the phase boundary remapping procedure is necessary before further analysis of the phase boundaries can be made. Indeed, in the simulations shown in the previous sections, the respective value of $\frac{\tau}{\tau_{\mathrm{D}}}$ is 1.54 . Therefore, the switching currents are much higher and the linear slope is different from that expected from the model. One also should notice that this formalism works only in high- $\Delta$ approximation. Therefore, the parts of the phase boundaries close to the regions where $H_{\text {ext }}$ approaches $H_{\perp}$ should be removed from the analysis.

From extrapolation of the voltage -driven boundaries to $\mathrm{V}=0$ one can estimate $H_{\perp} \sim 2.8-3.1 \mathrm{kOe}$, which is slightly higher than the corresponding value extracted from Eq. (10) (2.6 kOe). Nevertheless, the obtained $H_{\perp}$ values are in quite good agreement considering these two values are derived from very different physical phenomena (superparamagnetism vs STT switching). The spin-torque efficiency prefactor $s_{t \|}$ can be directly determined from the experimental slope using Eq. (9): $s_{t \|}=162 G_{p}^{-1} \mathrm{Oe} / \mathrm{V}$. From this, assuming that $G_{p}=1 / \mathrm{R} \times \mathrm{A}$, the effective spin polarization parameter in the system can be derived as $\eta=0.49$. If one uses the measured TMR value to estimate the polarization factor, assuming that $\eta=\sqrt{\mathrm{TMR}(\mathrm{TMR}+2)} /[2(\mathrm{TMR}+1)]$ [42] and $\mathrm{TMR}=1.26$, this would yield $\eta=0.44$, which is close to the value extracted from the diagram boundary slope. The zero-field switching current, recalculated using Eq. (8) for obtained values of $H_{\perp}, s_{t \|}$, and known parameter $\alpha$, gives $I_{\text {sw0 }}=0.35 G_{p} \mathrm{~V}$.

Therefore, one can conclude that the experiments carried out in the 36-nm pMTJ system can be well described within the macrospin approximation and thermally activated ballistic regime of STT switching. The $H_{\perp}, s_{t \|}$ parameters extracted from the phase boundaries of the $V_{\text {bias }}-H$ stability diagram are in good agreement with those extracted independently from the magnetoresistance loop and Néel-Brown model. It is worth noting that a macrospin behavior is not specific only to the measured device but is a generally observed feature for pillars with a nominal diameter $<80 \mathrm{~nm}$.

\section{LANDAU vs GILBERT}

In this section, we emphasize an important issue naturally arising from the analysis carried out in the previous sections. If the STT terms are added directly to the LL equation [43], then instead of Eq. (2) (obtained with the Gilbert dissipation term [44]), the following modified equation is obtained:

$$
\begin{aligned}
\frac{1}{\gamma \mu_{0}} \frac{d \hat{\boldsymbol{m}}}{d t}= & -\hat{\boldsymbol{m}} \times\left(\frac{1}{1+\alpha^{2}} \overrightarrow{\boldsymbol{H}}_{\mathrm{eff}}-a_{\perp} \hat{\boldsymbol{p}}\right)-\hat{\boldsymbol{m}} \\
& \times\left[\hat{\boldsymbol{m}} \times\left(\frac{\alpha}{1+\alpha^{2}} \overrightarrow{\boldsymbol{H}}_{\mathrm{eff}}+a_{\|} \hat{\boldsymbol{p}}\right)\right] .
\end{aligned}
$$

Still preserving the main features and general behavior of STT switching in fully perpendicular structures, Eq. (10) forbids switching only by the out-of-plane STT term, in contrast to Eq. (2), where the $\left[\alpha a_{\perp} \hat{\boldsymbol{m}} \times(\hat{\boldsymbol{m}} \times \hat{\boldsymbol{p}})\right]$ component allows the system to change its energy even if $a_{\|}=0$. That turns us to the still open discussion [45-52] of physical validity of Gilbert damping and Landau damping formulation in the magnetization dynamics equation. Although it is generally claimed that LL and LLG equations are mathematically equivalent, we can see a significant difference when the STT terms are added: the field-like STT term written in the LL equation is fully conservative, and it cannot change the system energy if Eq. (12) is chosen to describe the STT-induced dynamics. Leaving this fact "as is," one should notice that in numerical simulations, it is more common to use the LL form instead of the LLG form, and different ways to introduce STT terms [i.e., explicitly into the LL equation (Eq. 12) or via transformation of LLG + STT (Eq. 2)] can lead to significantly different results.

Figure 6 demonstrates this important issue by comparing examples of macrospin simulations using either LL or Gilbert damping terms to describe dissipation during STT-induced switching. Here, we adjusted the relative magnitudes of the field-like and damping-like STT prefactors to have comparable contributions in the second part of Eq. (2), which is the LLG + STT case. As soon as the field-like STT prefactor is set to have only a quadratic-bias voltage dependence (the case of a symmetrical tunnel junction), the produced torque always pulls the free layer magnetization in the antiparallel configuration with the fixed layer. The damping-like STT prefactor is set to be linear on the bias voltage, and therefore the torque direction is
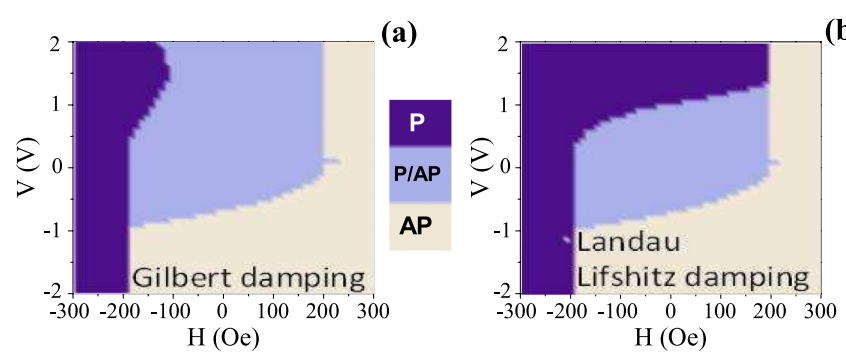

(b)

FIG. 6. (Color online) Two identical macrospin simulations of a stability phase diagram carried out at $T=0 \mathrm{~K}$ : (a) using Eq. (2), LLG + STT; (b) using Eq. (12), LL + STT. STT prefactors: $s_{t \|}=$ $12 G_{p}^{-1} \mathrm{Oe} / \mathrm{V}$ and $s_{t \perp 2}=400 G_{p}^{-2} \mathrm{Oe} / \mathrm{V}^{2}$. Other parameters are the same as used for the simulations in Sec. IV. 
determined by the current polarity. When a negative voltage is applied to the system, field-like torque helps the damping-like torque switch magnetization in the antiparallel state. It shifts the phase boundary toward lower switching voltages. However the expected boundary shift is too small to be visible in our simulations considering the chosen step for the voltage writing pulse amplitude. Also, a quadratic dependence of the field-like STT prefactor allows it to compete with the damping-like torque only at relatively high writing pulse voltages. At the same time, for positive pulses, field-like torque works against the damping-like torque, which shifts the phase boundary to higher voltages. The higher the switching voltage, the higher the relative contribution from the field-like torque. Finally, when the writing pulse is about $1.6 \mathrm{~V}$, field-like torque compensates the damping-like one, and further increase of the writing pulse amplitude starts shifting the phase boundary back toward negative fields, decreasing the field window of the bipolar STT switching. The same effect is observed at finite temperatures in Fig. 3(b) for the bottom middle diagram. This competition between the STT terms, however, is impossible in simulations with the Landau damping term because the $\alpha a_{\perp} \hat{\boldsymbol{m}} \times(\hat{\boldsymbol{m}} \times \hat{\boldsymbol{p}})$ term is absent in Eq. (12).

Finally, it is traditionally accepted that the LLG and LL equations are geometrically equivalent, and the mathematical transformation from one to another ends up with $\frac{1}{1+\alpha^{2}}$ rescaling of the gyromagnetic ratio. This $\frac{1}{1+\alpha^{2}}$ correction in real physical systems is very small and experimentally undetectable. However, this is not the case anymore if the STT terms are added to the LLG equation. The equations are now different. The same transformation (i.e., LLG + $\mathrm{STT} \rightarrow$ LL) leads to the appearance of two additional STT pseudo-torques $\left[\alpha a_{\perp} \hat{\boldsymbol{m}} \times(\hat{\boldsymbol{m}} \times \hat{\boldsymbol{p}}), \quad \alpha a_{\|} \hat{\boldsymbol{m}} \times \hat{\boldsymbol{p}}\right]$, which are linearly proportional to the damping constant $\alpha$ and in principle can be detected experimentally.

Experimentally, it should be possible to assess which formulation of damping is correct by measuring the variation of the precession frequency in the subswitching threshold regime in samples having various damping constants. Such samples could be produced, for instance, by depositing a wedge of Pt above the storage layer before the patterning of the wafer. For this experiment, it would be preferable to use symmetric MTJs so that the field-like torque has a quadratic dependence on bias voltage. If the LLG formulation is correct, we expect a linear variation of the frequency with damping constant under fixed bias voltage, whereas if the LL formulation is valid, no dependence of the frequency on damping should be observed.

\section{CONCLUSIONS}

It has been shown that the LLG equation with the field-like and damping-like STT terms transformed into the LL form considerably simplifies the analysis of the STT switching process. In the case of a fully perpendicular MTJ system, the boundaries of the $I-H$ stability phase diagram can be obtained directly from the transformed Eq. (2). It was shown that the field-like term has negligible influence on the STT switching process in pMTJs with low damping, influencing mainly the FMR precession frequency for the small oscillations near the equilibrium. Considering that in standard pMTJ structures its effective magnitude cannot be much higher than the magnitude of the in-plane torque, it would be hard to track its bias voltage (current) dependence from experimentally measured stability phase diagrams. Measuring the bias voltage dependence of the frequency in the precessional regime would certainly better reveal the influence of the field-like STT term, but the contribution of the field-like term still would have to be separated from the nonlinear influence of the oscillation amplitude on the frequency.

Finite temperature macrospin simulations in LLG-STT formalism under finite writing pulse duration have confirmed the negligible role of the field-like term in the STT switching process of pMTJ structure. Limitations of the macrospin model are not expected to be important in the case of pMTJ pillars with diameters comparable to or below the exchange length. This is confirmed by the experiments carried out on 36-nm-diameter pMTJ pillars.

One should note that the method developed for the phase boundaries construction gives the same results as those obtained from the analysis of dynamical response of the system carried out by different groups supposing the linear dependence of the damping-like STT prefactor versus applied bias voltage. However, we believe that it will be more useful in the interpretation of the experiment and simulations, because it is much more flexible, and it allows the introduction of any desirable current (voltage) dependences for the in-plane and out-of-plane STT prefactors.

Using the developed formalism, the spin-torque efficiency and effective spin polarization parameters have been derived from the current field stability diagram boundaries experimentally measured on a 36-nm pMTJ pillar. The obtained parameters have been cross-checked by estimations from magnetoresistance curves and from the thermally activated magnetization reversal regime. Good agreement between the values derived from the analysis of different physical principles strongly supports the assumption of macrospin behavior in the measured sample.

We also showed that the different dissipation terms (i.e., LL or Gilbert) give rise to different analytical expressions describing the phase boundaries of $I-H$ switching diagrams, which can be important in heavily damped systems. If the Landau damping term is physically correct, the action of the field-like and the damping-like torques in the pMTJ system is completely separated in precession and dissipation terms in the equation of dynamics. If the Gilbert damping term is correct, then two additional torques $\left[\alpha a_{\perp} \hat{\boldsymbol{m}} \times(\hat{\boldsymbol{m}} \times \hat{\boldsymbol{p}})\right.$ and $\left.\alpha a_{\|} \hat{\boldsymbol{m}} \times \hat{\boldsymbol{p}}\right]$ are mixed in with the main STT contributors $\left[a_{\|} \hat{\boldsymbol{m}} \times \hat{\boldsymbol{p}}\right.$ and $a_{\perp} \hat{\boldsymbol{m}} \times(\hat{\boldsymbol{m}} \times \hat{\boldsymbol{p}})$, respectively]. An experimental way to assess which damping formulation is correct in combination with STT was proposed.

\section{ACKNOWLEDGMENTS}

This paper was supported by the Samsung Global MRAM Innovation Program and EUROTALENTS Program. The authors are grateful to Ursula Ebels for fruitful discussions. 
[1] T. Liu, Y. Zhang, J. W. Cai, and H. Y. Pan, Sci. Rep. 4, 5895 (2014).

[2] A. V. Khvalkovskiy, D. Apalkov, S. Watts, R. Chepulskii, R. S. Beach, A. Ong, X. Tang, A. Driskill-Smith, W. H. Butler, P. B. Visscher, D. Lottis, E. Chen, V. Nikitin, and M. Krounbi, J. Phys. D: Appl. Phys. 46, 074001 (2013).

[3] S. Ikeda, K. Miura, H. Yamamoto, K. Mizunuma, H. D. Gan, M. Endo, S. Kanai, J. Hayakawa, F. Matsukura, and H. Ohno, Nat. Mater. 9, 721 (2010).

[4] N. Nishimura, T. Hirai, A. Koganei, T. Ikeda, K. Okano, Y. Sekiguchi, and Y. Osada, J. Appl. Phys. 91, 5246 (2002).

[5] A. D. Kent, Nat. Mater. 9, 699 (2010).

[6] B. Rodmacq, S. Auffret, B. Dieny, S. Monso, and P. Boyer, J. Appl. Phys. 93, 7513 (2003).

[7] M. Julliere, Phys. Lett. A 54, 225 (1975).

[8] W. Butler, Sci. Technol. Adv. Mater. 9, 014106 (2008).

[9] J. Mathon and A. Umerski, Phys. Rev. B 63, 220403(R) (2001).

[10] S. Monso, B. Rodmacq, S. Auffret, G. Casali, F. Fettar, B. Gilles, B. Dieny, and P. Boyer, Appl. Phys. Lett. 80, 4157 (2002).

[11] J. C. Slonczewski, J. Magn. Magn. Mater. 159, L1 (1996).

[12] L. Berger, Phys. Rev. B 54, 9353 (1996).

[13] J. Z. Sun, Phys. Rev. B 62, 570 (2000).

[14] J. A. Katine, F. J. Albert, R. A. Buhrman, E. B. Meyers, and D. C. Ralph, Phys. Rev. Lett. 84, 3149 (2000).

[15] K. Bernert, V. Sluka, C. Fowley, J. Lindner, J. Fassbender, and A. M. Deac, Phys. Rev. B 89, 134415 (2014).

[16] H. Liu, D. Bedau, J. Z. Sun, S. Mangin, E. E. Fullerton, J. A. Katine, and A. D. Kent, J. Magn. Magn. Mater. 358-359, 233 (2014).

[17] D. M. Apalkov and P. B. Visscher, Phys. Rev. B 72, 180405 (2005).

[18] H. Tomita, S. Miwa, T. Nozaki, S. Yamashita, T. Nagase, K. Nishiyama, E. Kitagawa, M. Yoshikawa, T. Daibou, M. Nagamine, T. Kishi, S. Ikegawa, N. Shimomura, H. Yoda, and Y. Suzuki, Appl. Phys. Lett. 102, 042409 (2013).

[19] R. H. Koch, J. A. Katine, and J. Z. Sun, Phys. Rev. Lett. 92, 088302 (2004).

[20] K. Yamada, K. Oomaru, S. Nakamura, T. Sato, and Y. Nakatani, Appl. Phys. Lett. 106, 042402 (2015).

[21] D. Pinna, A. D. Kent, and D. L. Stein, J. Appl. Phys. 114, 033901 (2013).

[22] J. Z. Sun, R. P. Robertazzi, J. Nowak, P. L. Trouilloud, G. Hu, D. W. Abraham, M. C. Gaidis, S. L. Brown, E. J. O'Sullivan, W. J. Gallagher, and D. C. Worledge, Phys. Rev. B 84, 064413 (2011).

[23] A. V. Silva, D. C. Leitao, H. Zhiwei, R. J. Macedo, R. Ferreira, E. Paz, F. L. Deepak, S. Cardoso, and P. P. Freitas, IEEE Trans. Magn. 49, 4405 (2013).

[24] D. V. Berkov and J. Miltat, J. Magn. Magn. Mater. 320, 1238 (2008).

[25] Y. Zhou, J. Åkerman, and J. Z. Sun, Appl. Phys. Lett. 98, 102501 (2011).

[26] C.-Y. You and M.-H. Jung, J. Appl. Phys. 113, 073904 (2013).

[27] C. Ortiz Pauyac, A. Kalitsov, A. Manchon, and M. Chshiev, Phys. Rev. B 90, 235417 (2014).

[28] A. Kalitsov, M. Chshiev, I. Theodonis, N. Kioussis, and W. H. Butler, Phys. Rev. B 79, 174416 (2009).
[29] I. Theodonis, N. Kioussis, A. Kalitsov, M. Chshiev, and W. H. Butler, Phys. Rev. Lett. 97, 237205 (2006).

[30] J. C. Sankey, Y. T. Cui, J. Z. Sun, J. C. Slonczewski, R. A. Buhrman, and D. C. Ralph, Nat. Phys. 4, 67 (2008).

[31] H. Kubota, A. Fukushima, K. Yakushiji, T. Nagahama, S. Yuasa, K. Ando, H. Maehara, Y. Nagamine, K. Tsunekawa, D. Djayaprawira, N. Watanabe, and Y. Suzuki, Nat. Phys. 4, 37 (2008).

[32] A. M. Deac, A. Fukushima, H. Kubota, H. Maehara, Y. Suzuki, S. Yuasa, Y. Nagamine, K. Tsunekawa, D. Djayaprawira, and N. Watanabe, Nat. Phys. 4, 803 (2008).

[33] A. Chanthbouala, R. Matsumoto, J. Grollier, V. Cros, A. Anane, A. Fert, A. V. Khvalkovskiy, K. A. Zvezdin, K. Nishimura, Y. Nagamine, H. Maehara, K. Tsunekawa, A. Fukushima, and S. Yuasa, Nat. Phys. 7, 626 (2011).

[34] S. C. Oh, S. Y. Park, A. Manchon, M. Chshiev, J. H. Han, H. W. Lee, J.-E. Lee, K.-T. Nam, Y. Jo, Y.-C. Kong, B. Dieny, and K. J. Lee, Nat. Phys. 5, 898 (2009).

[35] S. Petit, N. de Mestier, C. Baraduc, C. Thirion, Y. Liu, M. Li, P. Wang, and B. Dieny, Phys. Rev. B 78, 184420 (2008).

[36] S. Le Gall, J. Cucchiara, M. Gottwald, C. Berthelot, C.-H. Lambert, Y. Henry, D. Bedau, D. B. Gopman, H. Liu, A. D. Kent, J. Z. Sun, W. Lin, D. Ravelosona, J. A. Katine, Eric E. Fullerton, and S. Mangin, Phys. Rev. B 86, 014419 (2012).

[37] W. F. Brown, Jr., Phys. Rev. 130, 1677 (1963).

[38] J. L. García-Palacios and F. J. Lázaro, Phys. Rev. B 58, 14937 (1998).

[39] M. Hosomi, H. Yamagishi, T. Yamamoto, K. Bessho, Y. Higo, K. Yamane, H. Yamada, M. Shoji, H. Hachino, C. Fukumoto, H. Nagao, and H. Kano, Tech. Dig.--Int. Electron Devices Meet., 459 (2005).

[40] L. Néel, Ann. Geophys. (C. N. R. S.), 5, 99 (1949).

[41] T. Devolder, P. H. Ducrot, J. P. Adam, I. Barisic, N. Vernier, J. V. Kim, B. Ockert, and D. Ravelosona, Appl. Phys. Lett. 102, 022407 (2013).

[42] J. Z. Sun, S. L. Brown, W. Chen, E. A. Delenia, M. C. Gaidis, J. Harms, G. Hu, X. Jiang, R. Kilaru, W. Kula, G. Lauer, L. Q. Liu, S. Murthy, J. Nowak, E. J. O'Sullivan, S. S. P. Parkin, R. P. Robertazzi, P. M. Rice, G. Sandhu, T. Topuria, and D. C. Worledge, Phys. Rev. B 88, 104426 (2013).

[43] L. D. Landau and E. M. Lifshitz, Phys. Z. Sowjetunion 8, 153 (1935).

[44] T. L. Gilbert, IEEE Trans. Mag. 40, 3443 (2004).

[45] H. B. Callen, J. Phys. Chem. Solids 4, 256 (1958).

[46] M. D. Stiles, W. M. Saslow, M. J. Donahue, and A. Zangwill, Phys. Rev. B 75, 214423 (2007).

[47] H. J. Skadsem, Y. Tserkovnyak, A. Brataas, and G. E. W. Bauer, Phys. Rev. B 75, 094416 (2007).

[48] D. R. Fredkin and A. Ron, Phys. Rev. B 61, 8654 (2000).

[49] G. Bertotti, I. D. Mayergoyz, and C. Serpico, Physica B (Amsterdam, Neth.) 306, 102 (2001).

[50] A. Baral, S. Vollmar, and H. C. Schneider, Phys. Rev. B 90, 014427 (2014).

[51] W. M. Saslow, J. Appl. Phys. 105, 07D315 (2009).

[52] M. C. Hickey and J. S. Moodera, Phys. Rev. Lett. 102, 137601 (2009). 